\title{
Dielectric Relaxation of Lanthanide-Based Ternary Oxides: Physical and Mathematical Models
}

\author{
Chun Zhao, ${ }^{1,2}$ C. Z. Zhao, ${ }^{1,2,3}$ Jing Tao, ${ }^{3}$ M. Werner, ${ }^{2,4}$ S. Taylor, ${ }^{2}$ and P. R. Chalker ${ }^{4}$ \\ ${ }^{1}$ Department of Electrical and Electronic Engineering, Xi'an Jiaotong-Liverpool University, Suzhou 215123, China \\ ${ }^{2}$ Department of Electrical Engineering and Electronics, University of Liverpool, Liverpool L69 3GJ, UK \\ ${ }^{3}$ Department of Microelectronics, Xi'an Jiaotong University, Xi'an 710049, China \\ ${ }^{4}$ Department of Materials Science and Engineering, University of Liverpool, Liverpool L69 3GH, UK \\ Correspondence should be addressed to C.Z.Zhao, cezhou.zhao@xjtlu.edu.cn
}

Received 18 July 2011; Accepted 4 October 2011

Academic Editor: Shafiul Chowdhury

Copyright ( $) 2012$ Chun Zhao et al. This is an open access article distributed under the Creative Commons Attribution License, which permits unrestricted use, distribution, and reproduction in any medium, provided the original work is properly cited.

Cerium-doped hafnium oxides $\left(\mathrm{Ce}_{x} \mathrm{Hf}_{1-x} \mathrm{O}_{2}\right)$ and lanthanum-doped zirconium oxides $\left(\mathrm{La}_{x} \mathrm{Zr}_{1-x} \mathrm{O}_{2}\right)$ were investigated. The highest dielectric constants, $k$, were obtained from lightly doped oxides with an La content of $x=0.09$ and a Ce content of $x=0.1$, for which $k$-values of $33 \sim 40$ were obtained. The dielectric relaxation appears to be related to the size of crystal grains formed during annealing, which was dependent on the doping level. The physical and mathematical models were used to analyze the relationship between $k$-values and frequencies. The variations in the $k$-values up to megahertz frequencies for both $\mathrm{Ce}_{x} \mathrm{Hf}_{1-x} \mathrm{O}_{2}$ and $\mathrm{La}_{x} \mathrm{Zr}_{1-x} \mathrm{O}_{2}$ are simulated based on the Curie-von Schweidler (CS) or Havriliak-Negami (HN) relationships. Concerning the lightly doped $\mathrm{Ce}_{x} \mathrm{Hf}_{1-x} \mathrm{O}_{2}$ and $\mathrm{La}_{x} \mathrm{Zr}_{1-x} \mathrm{O}_{2}$, the data extracted are best modeled by the $\mathrm{HN}$ law, while $\mathrm{La}_{x} \mathrm{Zr}_{1-x} \mathrm{O}_{2}$ with doping level from $x=0.22$ to 0.63 are best modelled based on the CS law.

\section{Introduction}

As the thickness of SiON gate dielectric thin films used in complementary metal oxide semiconductor (CMOS) devices is reduced toward $1 \mathrm{~nm}$, the gate leakage current level becomes unacceptable [1]. To overcome this leakage problem, extensive efforts have been focused on finding alternative gate dielectrics for the $45 \mathrm{~nm}$ node and beyond technologies [2]. $\mathrm{ZrO}_{2}$ and $\mathrm{HfO}_{2}$ are two of the most promising high dielectric constant (high- $k$ ) materials for the replacement of $\mathrm{SiO}_{2}$ in MOSFETs, but it has been found that crystallization occurs at $\sim 500^{\circ} \mathrm{C}$ for pure hafnia [3]. Doping with lanthanum $(\mathrm{La})$ or cerium $(\mathrm{Ce})$ increases the crystallization temperature [4]. Doping hafnia and zirconia thin films with rare earth elements can also stabilize the metastable tetragonal or cubic phase following annealing which enhances the dielectric constant [5]. Since dielectric relaxation and the associated losses can impair MOSFET performance, the larger dielectric relaxation of most high- $k$ dielectrics compared to $\mathrm{SiO}_{2}$ is a significant issue for their use [6].
Capacitance-voltage $(C-V)$ measurements are a fundamental characterization technique for MOS devices. $\mathrm{C}-\mathrm{V}$ measurements have been widely used to extract the dielectric constant ( $k$-value) and dielectric loss of high- $k$ materials using the capacitance measured in strong accumulation. Several models and analytical formulae have been thoroughly investigated for correcting the data from frequency dispersion [7-11]. When the above effects were taken into account, frequency dispersion in the accumulation region of $\mathrm{C}-\mathrm{V}$ curves is still observed from high- $k$ dielectric thin films due to the decrease of $k$-value with frequency $(f)$, which is identified as a dielectric relaxation effect $[12,13]$.

However, there is insufficient information about dielectric relaxation of high- $k$ thin films, which prompts us to investigate the phenomenon and the underlying mechanism. In this paper, the dielectric relaxation and frequency dependence of the $k$-value of $\mathrm{Ce}_{x} \mathrm{Hf}_{1-x} \mathrm{O}_{2}$ and $\mathrm{La}_{x} \mathrm{Zr}_{1-x} \mathrm{O}_{2}$ dielectrics were examined. The physical and mathematical models used were according to recent research for fitting the measured $k-f$ curves. Variations in the $k$-value up to 
megahertz frequencies are simulated based on the Curie-von Schweidler (CS) or Havriliak-Negami (HN) relationships.

\section{Device Preparation and Measurements}

Amorphous $\mathrm{La}_{x} \mathrm{Zr}_{1-x} \mathrm{O}_{2}$ thin films $(x=0.09,0.22,0.35$, $0.63)$ and $\mathrm{Ce}_{x} \mathrm{Hf}_{1-x} \mathrm{O}_{2}$ thin films $(x=0.1)$ were deposited on $n$-type $\mathrm{Si}(100)$ substrates using liquid injection atomic layer deposition (ALD), carried out on an Aixtron AIX 200FE AVD reactor fitted with the "Trijet" liquid injector system. The doping level was varied up to a concentration level of $63 \%$, that is, $x=0.63$. The interfacial layer between high- $k$ thin film and silicon substrate is a $\sim 1 \mathrm{~nm}$ native $\mathrm{SiO}_{2}$ determined by cross-section transmission electron microscopy (XTEM). Samples were then annealed at $900^{\circ} \mathrm{C}$ for 15 minutes in an $\mathrm{N}_{2}$ ambient to crystallize the thin films.

All the MOS capacitors were fabricated by thermal evaporation of Au gates through a shadow mask with an effective area of $4.9 \times 10^{-4} \mathrm{~cm}^{2}$. The backside contact of the Si wafers was cleaned with a buffer HF solution and subsequently a $200 \mathrm{~nm}$ thickness of Al was deposited by thermal evaporation to minimize the effect of series resistance. The physical properties of the thin films were studied using auger electron spectroscopy, X-ray diffraction, medium energy ion scattering, XTEM, and atomic force microscopy. Their electrical properties were investigated by current-voltage $(I-V)$, high-frequency capacitance-voltage $(C-V)$, and capacitancefrequency $(C-f)$ measurements.

\section{Experimental Results and Discussion}

Typical $C$ - $V$ results of $\mathrm{SiO}_{2}, \mathrm{HfO}_{2}$, and $\mathrm{Ce}_{0.1} \mathrm{Hf}_{0.9} \mathrm{O}_{2}$ dielectrics are shown in Figure 1 and Figure 2, respectively. The $C-V$ results shown in Figure 1 are taken from a reference $\mathrm{SiO}_{2}$ sample (Figure 1(a)) and from an $\mathrm{HfO}_{2}$ sample (Figure 1(b)). No significant variation in $\mathrm{C}-\mathrm{V}$ response was observed in the reference $\mathrm{SiO}_{2}$ and $\mathrm{HfO}_{2}$ dielectrics, which confirms that the $C-V$ measurement system does not introduce any frequency dispersion. Furthermore, Figure 1(b) shows that the interfacial layer $\left(\sim 1 \mathrm{~nm}\right.$ native $\left.\mathrm{SiO}_{2}\right)$ between the $\mathrm{HfO}_{2}$ and the Si substrate also introduces negligible frequency dispersion for the samples used here $[9,11]$.

However, for the various frequencies ranging from $100 \mathrm{~Hz}$ to $200 \mathrm{kHz}$ applied on the annealed $x=0.1$ sample of $\mathrm{Ce}_{x} \mathrm{Hf}_{1-x} \mathrm{O}_{2}$ thin films, different $C-V$ results are exhibited, especially obtained in strong accumulation (unlike the $\mathrm{SiO}_{2}$ and $\mathrm{HfO}_{2}$ in Figure 1). Figure 2 shows that substantial frequency dispersion was observed during $C$ - $V$ measurements on the $\mathrm{Ce}_{x} \mathrm{Hf}_{1-x} \mathrm{O}_{2}$ sample. The results of Figure 2 demonstrate that frequency dispersion can occur occasionally regardless of the interfacial layer of MOS structures and measurement system. In this case, the frequency dependence of the $k$-value is due to dielectric relaxation. The dielectric relaxation is modelled in more detail in follow, using the CS and Kohlrausch-Williams-Watts (KWW) or HN law to accurately simulate the measured phenomena.

The dielectric relaxation in the time domain can be described by a power-law time dependence (CS law), $t^{-n}$, or a stretched exponential time dependence (KWW law),

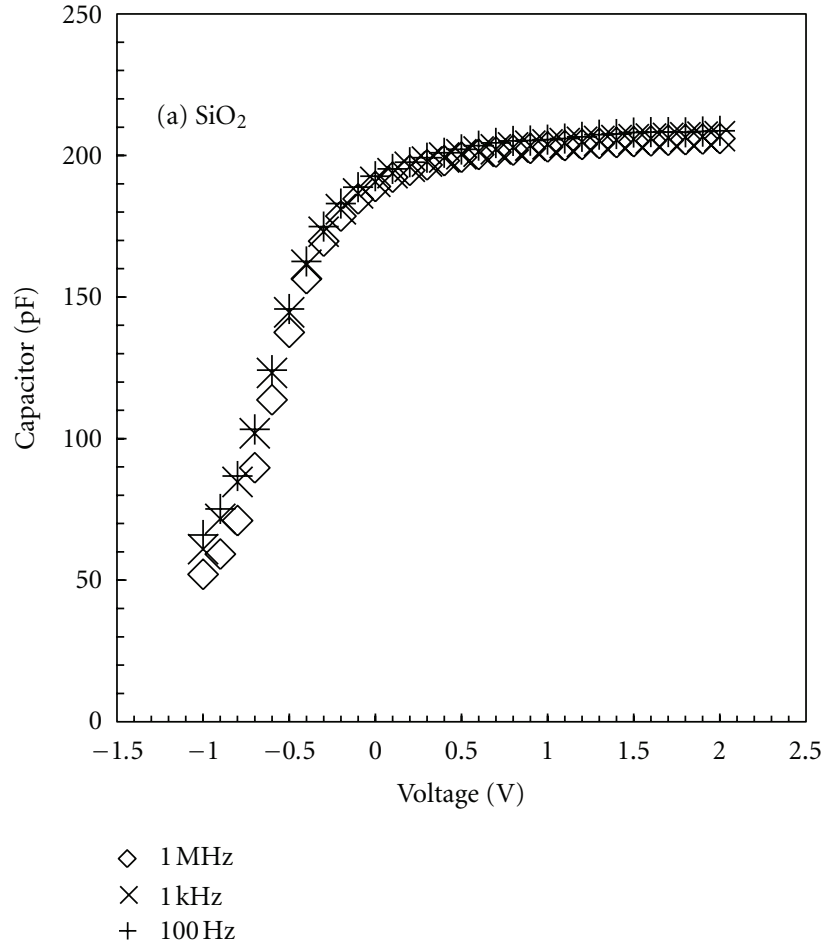

(a)

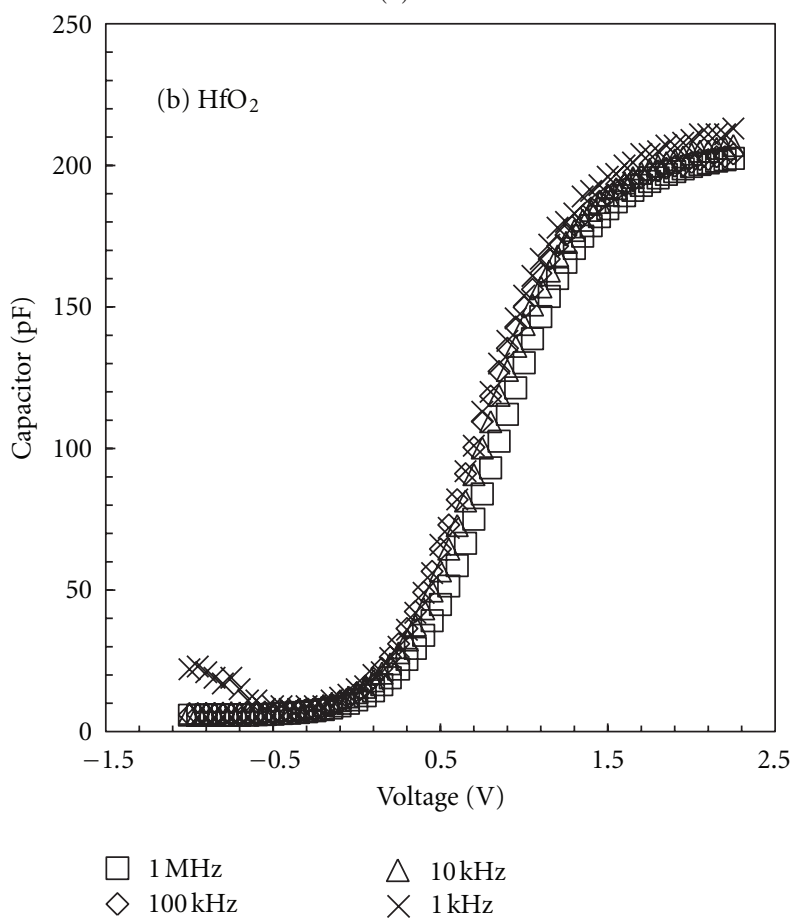

(b)

Figure 1: $C$ - $V$ measurements from $\mathrm{SiO}_{2}$ (a) and $\mathrm{HfO}_{2}$ (b) at different frequencies. No significant frequency response was observed in the reference $\mathrm{SiO}_{2}$ and $\mathrm{HfO}_{2}$ dielectrics.

$\exp \left[-\left(t / t_{0}\right)^{m}\right]$, where $n$ and $m$ are parameters ranging between 0 and 1 and $t_{0}$ is a characteristic relaxation time [1417]. There exist two alternative physical approaches to the interpretation of dielectric relaxation: the parallel and series models [18]. 


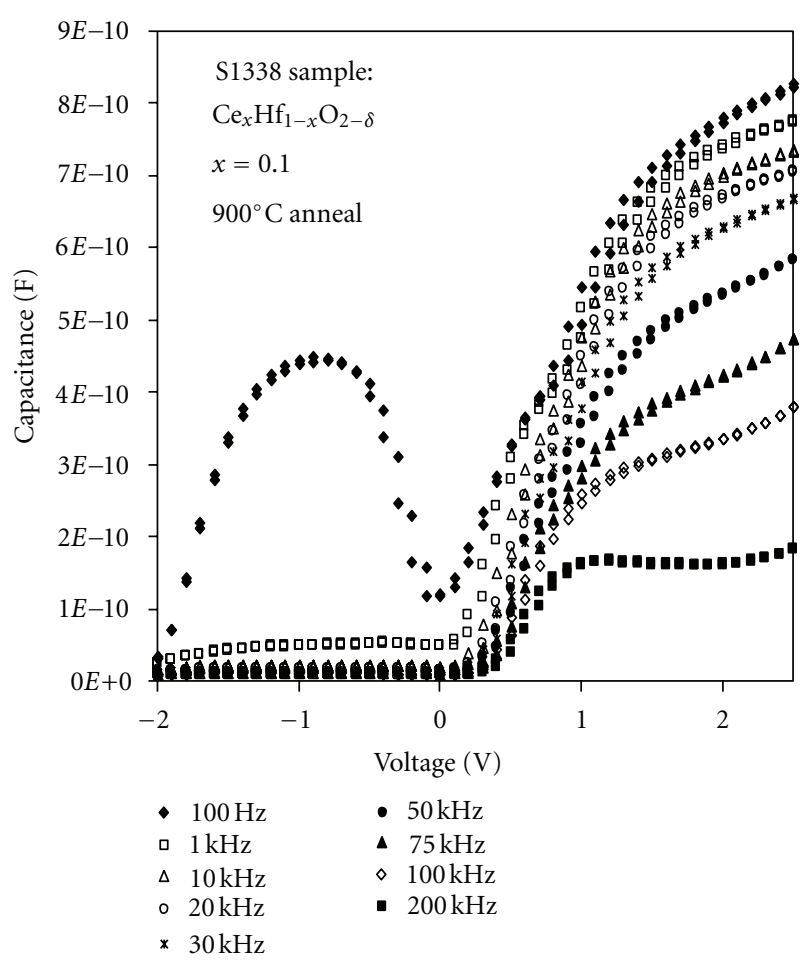

Figure 2: $C-V$ measurements from a $\mathrm{Ce}_{x} \mathrm{Hf}_{1-x} \mathrm{O}_{2}$ thin film at different frequencies (from $100 \mathrm{~Hz}$ to $200 \mathrm{kHz}$ ). Frequency dispersion was obtained regardless of the interfacial layer of MOS structures and measurement system, which was identified due to the frequency dependence of $k$-value (termed dielectric relaxation).

The parallel model represents the classical relaxation of a large assembly of individual relaxing entities such as dipoles, each of which relaxes with an exponential probability but has a different relaxation time $t_{k}[17,18]$. The total relaxation process corresponds to a summation over the available modes $k$, given a frequency domain response function. Parallel processes are possible among independent noninteracting entities. The parallel model may be approximated by the HN relationship. The alternative approach is the series model, whereby each successive stage of relaxation is conditioned by the immediate past history. The series model may be used to describe briefly the origins of the CS law (the $t^{-n}$ behavior).

Dielectric relaxation can be modeled by either the CS law or the HN law (KWW law) for high- $k$ thin films. However, in some complex condensed systems, neither the pure parallel nor the pure series approach is accepted and instead observed results interpolate smoothly between these extremes [19]. The CS behavior is verified to be faster than the $\mathrm{HN}$ function at short times and slower than the HN function at long times.

The general type of dielectric relaxation can be described from the CS law and the KWW law which are formulated as follows:

$$
\begin{gathered}
\frac{d P_{\mathrm{CS}}}{d t} \propto t^{-n} \quad \text { with } 0 \leq n \leq 1, \\
P_{\mathrm{KWW}} \propto \exp \left[-\left(\frac{t}{\tau}\right)^{\beta_{0}}\right] \quad \text { with } 0 \leq \beta_{0} \leq 1,
\end{gathered}
$$

where $P_{\mathrm{CS}}$ is the CS polarization and the exponent $n$ indicates the degree of dielectric relaxation. $P_{\mathrm{KWW}}$ is the KWW polarization, $\tau$ is the KWW relaxation time, and $\beta_{0}$ is the $P_{\mathrm{KWW}}$ parameter. The complex susceptibilities, $\chi_{\mathrm{CS}}$ and $\chi_{\mathrm{KWW}}$ (frequency domain), related to the CS law and KWW law, are simply the Fourier transforms of the above time-domain responses [20]:

$$
\chi_{\mathrm{CS}}(\omega) \propto F\left(\frac{d P_{\mathrm{CS}}}{d t}\right) \propto \int_{0}^{\infty} t^{-n} \exp (-i \omega t) d t \propto(i \omega)^{n-1}
$$

or

$$
\chi_{\mathrm{CS}}=A(i \omega)^{n-1},
$$

where $n$ and $A$ are the relaxation parameters, and

$$
\begin{aligned}
\chi_{\mathrm{KWW}}(\omega) \propto F( & \left.\frac{d P_{\mathrm{KWW}}}{d t}\right) \propto \int_{0}^{\infty} \frac{\beta_{0}}{\tau}\left(\frac{t}{\tau}\right)^{\beta_{0}-1} \\
& \times \exp \left[-\left(\frac{t}{\tau}\right)^{\beta_{0}}\right] \exp (-i \omega t) d t .
\end{aligned}
$$

In the frequency domain, after a Fourier transform, the corresponding dielectric response (3) can be approximated by a simple HN relationship:

$$
\chi_{\mathrm{HN}}(\omega)=\frac{\left(\varepsilon_{s}-\varepsilon_{\infty}\right)}{\left(1+(i \omega \tau)^{1-\alpha}\right)^{\beta_{1}}},
$$

where $\varepsilon_{s}$ and $\varepsilon_{\infty}$ are the static and high-frequency limit permittivities, respectively, $\omega=2 \pi f$ is the angular frequency, the $\tau$ is the HN relaxation time, and $a$ and $\beta_{1}$ are the HN relaxation parameters. The parameter $\alpha$ is a distribution parameter, which accounts for the decrease of the peak loss and broadening of the distribution. This was also used in the Cole-Cole equation [18].

The reason for replacing the KWW law using the HN relationship is to avoid the complicated numerical calculation implicit in (4). It was reported by Bokov and Ye that any Fourier transform of the KWW function into the frequency domain can be approximated by an HN function, but not vice versa [20].

Then the intrinsic effect of frequency dispersion caused by the dielectric relaxation can be described by the CS law (3) and/or the HN relationship (5) [21, 22]. Separating the real and imaginary parts of the CS equation (3) gives the following equation for $\varepsilon^{\prime}$ and $\varepsilon^{\prime \prime}$ [23]:

$$
\begin{gathered}
\mathcal{\varepsilon}^{\prime}(\omega)=\varepsilon_{\infty}+B \omega^{n-1}, \\
\mathcal{\varepsilon}^{\prime \prime}(\omega)=C \omega^{n-1},
\end{gathered}
$$




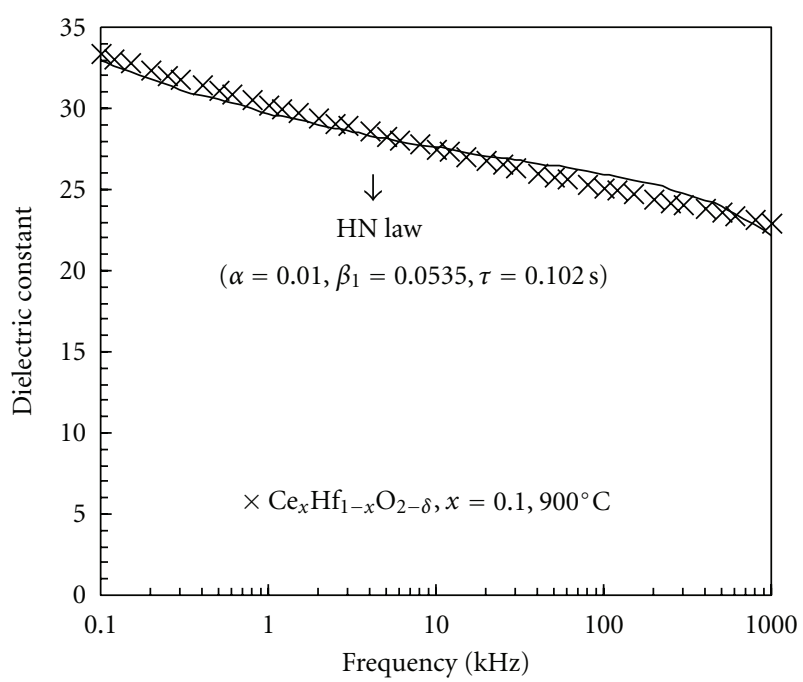

Figure 3: Dielectric relaxation of $\mathrm{Ce}_{x} \mathrm{Hf}_{1-x} \mathrm{O}_{2}$ observed in $C-V$ measurements (Figure 2) can be fitted by the $\mathrm{HN}$ relationship. The cross-symbols are the measured data and the solid line is from HN relationship given by $(7)$.

where $B$ and $C$ are constants. The real and imaginary parts of $\mathrm{HN}$ equation (5) may be separated which gives the following equation for $\varepsilon^{\prime}$ and $\varepsilon^{\prime \prime}[24]$ :

$$
\begin{aligned}
\mathcal{E}^{\prime}(\omega)= & \varepsilon_{\infty}+\left(\varepsilon_{s}-\varepsilon_{\infty}\right) \\
& \times \frac{\cos \beta_{1} \varphi}{\left[1+2\left(\omega \tau_{o}\right)^{1-\alpha} \sin (1 / 2) \alpha \pi+\left(\omega \tau_{o}\right)^{2(1-\alpha)}\right]^{\beta_{1} / 2}},
\end{aligned}
$$

$$
\begin{aligned}
\mathcal{\varepsilon}^{\prime \prime}(\omega)= & \varepsilon_{\infty}+\left(\varepsilon_{s}-\varepsilon_{\infty}\right) \\
& \times \frac{\sin \beta_{1} \varphi}{\left[1+2\left(\omega \tau_{o}\right)^{1-\alpha} \sin (1 / 2) \alpha \pi+\left(\omega \tau_{o}\right)^{2(1-\alpha)}\right]^{\beta_{1} / 2}},
\end{aligned}
$$

where

$$
\varphi=\operatorname{arctg}\left[\frac{\left(\omega \tau_{o}\right)^{1-\alpha} \cos (1 / 2) \pi \alpha}{1+2\left(\omega \tau_{o}\right)^{1-\alpha} \sin (1 / 2) \alpha \pi}\right] .
$$

The measured relationship between the $k$-value and frequency can be extracted from Figure 2, as shown in Figure 3 (cross-symbols). The experimentally observed dielectric relaxation for $\mathrm{Ce}_{x} \mathrm{Hf}_{1-x} \mathrm{O}_{2}$ was modeled using the $\mathrm{HN}$ relationship (7), as shown in Figure 3 (solid line); that is, the cross-symbols in Figure 3 are extracted from the measured data and the solid line is from the model using the $\mathrm{HN}$ relationship (7). For the sample used $\left(\mathrm{Ce}_{x} \mathrm{Hf}_{1-x} \mathrm{O}_{2}\right.$, the composition of Ce is $x=0.10$ ), the relaxation parameter $\alpha, \beta_{1}$, and the relaxation time $\tau$ are $0.01,0.0535$, and $0.102 \mathrm{~s}$, respectively. The fitted result, indicated by the solid line, is very close to the measured data.

For comparison to the $\mathrm{Ce}_{x} \mathrm{Hf}_{1-x} \mathrm{O}_{2}$ results shown in Figure 3, the $k-f$ data from the $\mathrm{La}_{x} \mathrm{Zr}_{1-x} \mathrm{O}_{2}$ thin films are shown in Figure 4 . The square symbols are the measured data

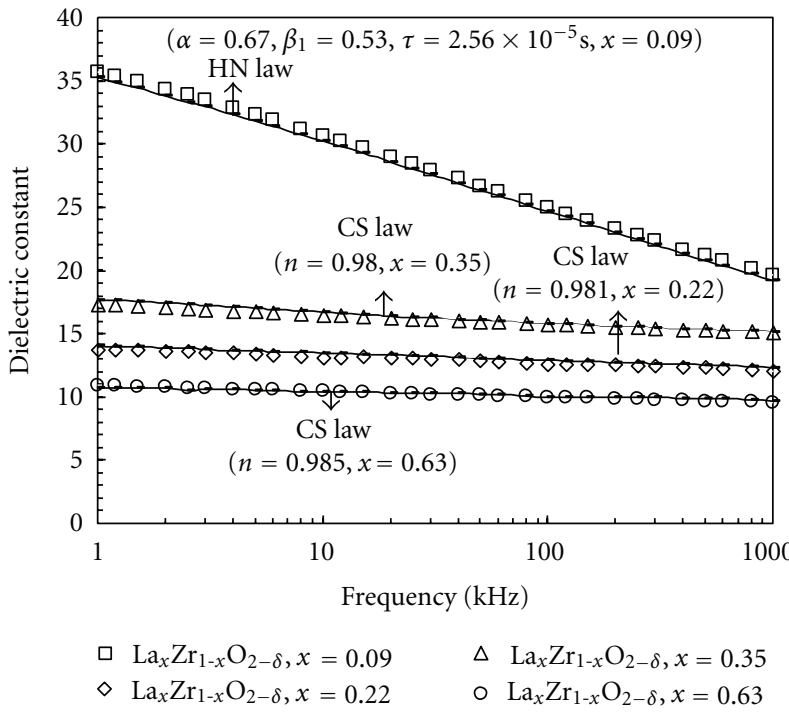

Figure 4: $k-f$ data taken from the measurement $C-f$ curves of $\mathrm{La}_{x} \mathrm{Zr}_{1-x} \mathrm{O}_{2}$ thin films. The square symbols are measured from $\mathrm{La}_{0.09} \mathrm{Zr}_{0.91} \mathrm{O}_{2}$, and the solid line is modeled according to the $\mathrm{HN}$ law. The diamond symbols, triangle symbols, and circle symbols are tested, respectively, from the $\mathrm{La}_{x} \mathrm{Zr}_{1-x} \mathrm{O}_{2}$ with doping levels of $22 \%$, $35 \%$, and $63 \%$, and solid lines in these cases are all simulated using the CS law. The parameters of CS and HN laws $(\alpha, \tau$, and $n)$ are shown in brackets.

from $\mathrm{La}_{0.09} \mathrm{Zr}_{0.91} \mathrm{O}_{2}$ where the solid line is modeled from the $\mathrm{HN}$ law. The diamond symbols, triangle symbols, and circle symbols are experimental data from the $\mathrm{La}_{x} \mathrm{Zr}_{1-x} \mathrm{O}_{2}$ thin films with doping levels of $22 \%, 35 \%$, and $63 \%$, where the solid lines are all simulated using the CS law. The parameters $(\alpha, \tau$, and $n)$ used are also listed in the figure.

Figure 4 summarizes the frequency dependence of $k$ values for different La concentrations $x$. The zirconia thin film with an La concentration of $x=0.35$ had a relatively flat frequency response and a $k$-value of $\sim 17$ (on average). In the same way the La-doped zirconium oxides with doping level of $22 \%$ and $63 \%$ showed average values of 14 and 11 , respectively. In contrast the lightly doped sample (9\%) had a substantially increased $k$-value but suffered from a more severe dielectric relaxation. A $k$-value of 35 was obtained at $1 \mathrm{kHz}$, but this value was found to reduce to a $k$-value of 25 at $1 \mathrm{MHz}$.

The $k$ - $f$ relationship of the $\mathrm{La}_{x} \mathrm{Zr}_{1-x} \mathrm{O}_{2}$ with the doping level of $9 \%$ is better modeled by the HN law (rather than the CS law), and the relaxation parameter $\alpha, \beta_{1}$, and the relaxation time $\tau$ are $0.67,0.53$, and $2.56 \times 10^{-5} \mathrm{~s}$, respectively. The $k$-values of the $\mathrm{La}_{x} \mathrm{Zr}_{1-x} \mathrm{O}_{2}$ dielectrics $(x=0.22,0.35$, and 0.63 ) clearly show power-law dependence on frequency $(f)$ and may be modeled using the CS law, $k \propto f^{n-1}(0 \leq$ $n \leq 1)$, where the value of the exponent $(n)$ indicates the degree of dielectric relaxation [6]. $n$ values of $0.981,0.98$, and 0.985 are obtained for La compositions $(x)$ of $0.22,0.35$, and 0.63 , respectively. Comparing $n$ values to $k$-values, it seems that the larger $k$-value suffers from more severe dielectric relaxation (smaller $n$ value). The largest $k$-value appears in $\mathrm{La}_{0.09} \mathrm{Zr}_{0.91} \mathrm{O}_{2}$, but the most severe dielectric relaxation 
occurs, and this could be directly related to the size of crystal grains formed during annealing [25].

Considering the lightly doped high- $k$ thin films $\left(\mathrm{Ce}_{x} \mathrm{Hf}_{1-x} \mathrm{O}_{2}\right.$ and $\left.\mathrm{La}_{x} \mathrm{Zr}_{1-x} \mathrm{O}_{2}\right)$, the extracted data are both best modeled by the HN law, which show with significant permittivity enhancement accompanied by a more pronounced dielectric relaxation.

For the $\mathrm{Ce}_{x} \mathrm{Hf}_{1-x} \mathrm{O}_{2}$ and $\mathrm{La}_{x} \mathrm{Zr}_{1-x} \mathrm{O}_{2}$ dielectrics possible causes for the observed dielectric relaxation have been suggested [26]. It has been reported that a decrease in crystal grain size can cause an increase in the dielectric relaxation in ferroelectric relaxor ceramics $[27,28]$. In addition to the doping level affecting the phase that the thin films crystallize during annealing, the doping level also affects the size of the crystal grains formed. It is possible therefore that the dielectric relaxation behavior observed in Figures 3 and 4 is due to the level of mechanical stress in the crystalline grains, which depends on the grain size, as has been found in the case of ferroelectric ceramics [23].

\section{Conclusion}

Doping hafnia and zirconia thin films with rare earth elements can stabilize the metastable tetragonal or cubic phase following annealing which enhances the dielectric constant. The level of enhancement is closely related to the doping level. Dielectric constants of 39 and 33 were obtained for $\mathrm{La}_{0.09} \mathrm{Zr}_{0.91} \mathrm{O}_{2}$ and $\mathrm{Ce}_{0.1} \mathrm{Hf}_{0.9} \mathrm{O}_{2}$ thin films, respectively. After taking into account the effect of the measurement system and the interfacial layer between the high- $k$ dielectrics and silicon substrate, the change of the real permittivity with frequency has been modeled by either the CS or HN relationships. Comprehensive physical and mathematical models are provided and discussed for modelling the dielectric relaxation behaviour of high- $k$ thin films under various conditions. For the lightly doped high- $k$ thin films $\left(\mathrm{Ce}_{0.1} \mathrm{Hf}_{0.9} \mathrm{O}_{2}\right.$ and $\left.\mathrm{La}_{0.09} \mathrm{Zr}_{0.91} \mathrm{O}_{2}\right)$, the measured data are both best modeled by the HN expression with significant permittivity enhancement and serious dielectric relaxation. The lanthanum-doped zirconium oxides $\left(\mathrm{La}_{x} \mathrm{Zr}_{1-x} \mathrm{O}_{2}\right)$ with doping level from $22 \%, 35 \%$, to $63 \%$ are best modeled based on the CS law in comparison with lightly doped $\mathrm{La}_{x} \mathrm{Zr}_{1-x} \mathrm{O}_{2}$. Films with larger $k$-values suffer from more severe dielectric relaxation, which is probably related to the size of crystal grains formed during annealing.

\section{Acknowledgments}

This research was funded in part from the National Natural and Science Foundation of China under Grant no. 60976075 and from the Suzhou Science and Technology Bureau of China under Grant SYG201007.

\section{References}

[1] N. Lu, H. J. Li, J. J. Peterson, and D. L. Kwong, "HfTiAlO dielectric as an alternative high- $k$ gate dielectric for the next generation of complementary metal-oxide-semiconductor devices," Applied Physics Letters, vol. 90, no. 8, Article ID 082911, 2007.

[2] T. S. Böscke, S. Govindarajan, C. Fachmann et al., “Tetragonal phase stabilization by doping as an enabler of thermally stable $\mathrm{HfO}_{2}$ based MIM and MIS capacitors for sub 50nm deep trench DRAM," in Proceedings of the International Electron Devices Meeting (IEDM '06), San Francisco, Calif, USA, 2006.

[3] Y. Yamamoto, K. Kita, K. Kyuno, and A. Toriumi, "Structural and electrical properties of $\mathrm{HfLaO}_{x}$ films for an amorphous high- $k$ gate insulator," Applied Physics Letters, vol. 89, no. 3, Article ID 032903, 3 pages, 2006.

[4] S. V. Ushakov, C. E. Brown, and A. Navrotsky, "Effect of La and $\mathrm{Y}$ on crystallization temperatures of hafnia and zirconia," Journal of Materials Research, vol. 19, no. 3, pp. 693-696, 2004.

[5] B. Mereu, A. Dimoulas, G. Vellianitis, G. Apostolopoulos, R. Scholz, and M. Alexe, "Interface trap density in amorphous La $2 \mathrm{Hf} 2 \mathrm{O} 7 / \mathrm{SiO} 2$ high- $k$ gate stacks on Si," Applied Physics A: Materials Science and Processing, vol. 80, no. 2, pp. 253-257, 2005.

[6] B. Lee, T. Moon, T.-G. Kim, D.-K. Choi, and B. Park, "Dielectric relaxation of atomic-layer-deposited $\mathrm{HfO}_{2}$ thin films from $1 \mathrm{kHz}$ to $5 \mathrm{GHz}$," Applied Physics Letters, vol. 87, no. 1, pp. 1-3, 2005.

[7] E. H. Nicollian and J. R. Brews, MOS (Metal Oxide Semiconductor) Physics and Technology, Wiley Classic Library, 2003.

[8] K. S. K. Kwa, S. Chattopadhyay, N. D. Jankovic, S. H. Olsen, L. S. Driscoll, and A. G. O’Neill, "A model for capacitance reconstruction from measured lossy MOS capacitance-voltage characteristics," Semiconductor Science and Technology, vol. 18, no. 2, pp. 82-87, 2003.

[9] R. Rios, N. D. Arora, and C. L. Huang, "Analytic polysilicon depletion effect model for MOSFET's," IEEE Electron Device Letters, vol. 15, no. 4, pp. 129-131, 1994.

[10] A. S. Spinelli, A. Pacelli, and A. L. Lacaita, "An improved formula for the determination of the polysilicon doping," IEEE Electron Device Letters, vol. 22, no. 6, pp. 281-283, 2001.

[11] J. L. Zhang, J. S. Yuan, Y. Ma, and A. S. Oates, "Modeling of direct tunneling and surface roughness effects on $\mathrm{C}-\mathrm{V}$ characteristics of ultra-thin gate MOS capacitors," Solid-State Electronics, vol. 45, no. 2, pp. 373-377, 2001.

[12] C. Dubourdieu, E. Rauwel, C. Millon et al., "Growth by liquidinjection MOCVD and properties of $\mathrm{HfO}_{2}$ films for microelectronic applications," Chemical Vapor Deposition, vol. 12, no. 2-3, pp. 187-192, 2006.

[13] T.-M. Pan, J.-D. Lee, and W.-W. Yeh, "Influence of oxygen content on the structural and electrical characteristics of thin neodymium oxide gate dielectrics," Journal of Applied Physics, vol. 101, no. 2, Article ID 024110, 2007.

[14] J. Curie, "Recherches sur le pouvoir inducteur specifique et sur la conductibilite des corps cristallises," Annual Review of Physical Chemistry, vol. 18, p. 203, 1889.

[15] E. Von Schweidler, "Studien uber die anomalien im verhalten der dielektrika," Annalen der Physik, vol. 329, no. 14, pp. 711770, 1907.

[16] F. Kohlrausch, "Ueber die elastische Nachwirkung bei der Torsion," Poggendorff's Annalen der Physik, vol. 195, no. 7, pp. 337-368, 1863.

[17] G. Williams and D. C. Watts, "Non-symmetrical dielectric relaxation behaviour arising from a simple empirical decay function," Transactions of the Faraday Society, vol. 66, pp. 8085, 1970.

[18] A. K. Jonscher, Universal Relaxation Law-A sequel to Dielectric Relaxation in Solids, Chelsea Dielectrics Press, 1996. 
[19] A. Hunt, "Approximate power-law conductivity in the multiple-hopping regime," Journal of Non-Crystalline Solids, vol. 183, no. 1-2, pp. 109-121, 1995.

[20] A. A. Bokov and Z.-G. Ye, "Double freezing of dielectric response in relaxor $\mathrm{Pb}\left(\mathrm{Mg}_{1 / 3} \mathrm{Nb}_{2 / 3}\right) \mathrm{O}_{3}$ crystals," Physical Review B, vol. 74, no. 13, Article ID 132102, 2006.

[21] X. Zhao and D. Vanderbilt, "First-principles study of structural, vibrational, and lattice dielectric properties of hafnium oxide," Physical Review B, vol. 65, no. 23, Article ID 233106, pp. 1-4, 2002.

[22] D. Fischer and A. Kersch, "The effect of dopants on the dielectric constant of $\mathrm{HfO}_{2}$ and $\mathrm{ZrO}_{2}$ from first principles," Applied Physics Letters, vol. 92, no. 1, Article ID 012908, 2008.

[23] L. C. Costa and F. Henry, "Dielectric universal law of lead silicate glasses doped with neodymium oxide," Journal of NonCrystalline Solids, vol. 353, no. 47-51, pp. 4380-4383, 2007.

[24] F. Prégaldiny, C. Lallement, R. Van Langevelde, and D. Mathiot, "An advanced explicit surface potential model physically accounting for the quantization effects in deep-submicron MOSFETs," Solid-State Electronics, vol. 48, no. 3, pp. 427-435, 2004.

[25] H. Choosuwan, R. Guo, A. S. Bhalla, and U. Balachandran, "Low-temperature dielectric behavior of $\mathrm{Nb}_{2} \mathrm{O}_{5}-\mathrm{SiO}_{2}$ solid solutions," Journal of Applied Physics, vol. 93, no. 5, pp. 28762879, 2003.

[26] C. Z. Zhao, S. Taylor, M. Werner et al., "Dielectric relaxation of lanthanum doped zirconium oxide," Journal of Applied Physics, vol. 105, no. 4, Article ID 044102, 2009.

[27] C. Z. Zhao, S. Taylor, M. Werner et al., "high- $k$ materials and their response to gamma ray radiation," Journal of Vacuum Science and Technology B, vol. 27, no. 1, pp. 411-415, 2009.

[28] C. Z. Zhao, M. Werner, S. Taylor, P. R. Chalker, A. C. Jones, and C. Zhao, "Dielectric relaxation of La-doped Zirconia caused by annealing ambient," Nanoscale Research Letters, vol. 6, no. 1 , article 4, pp. 1-6, 2011. 

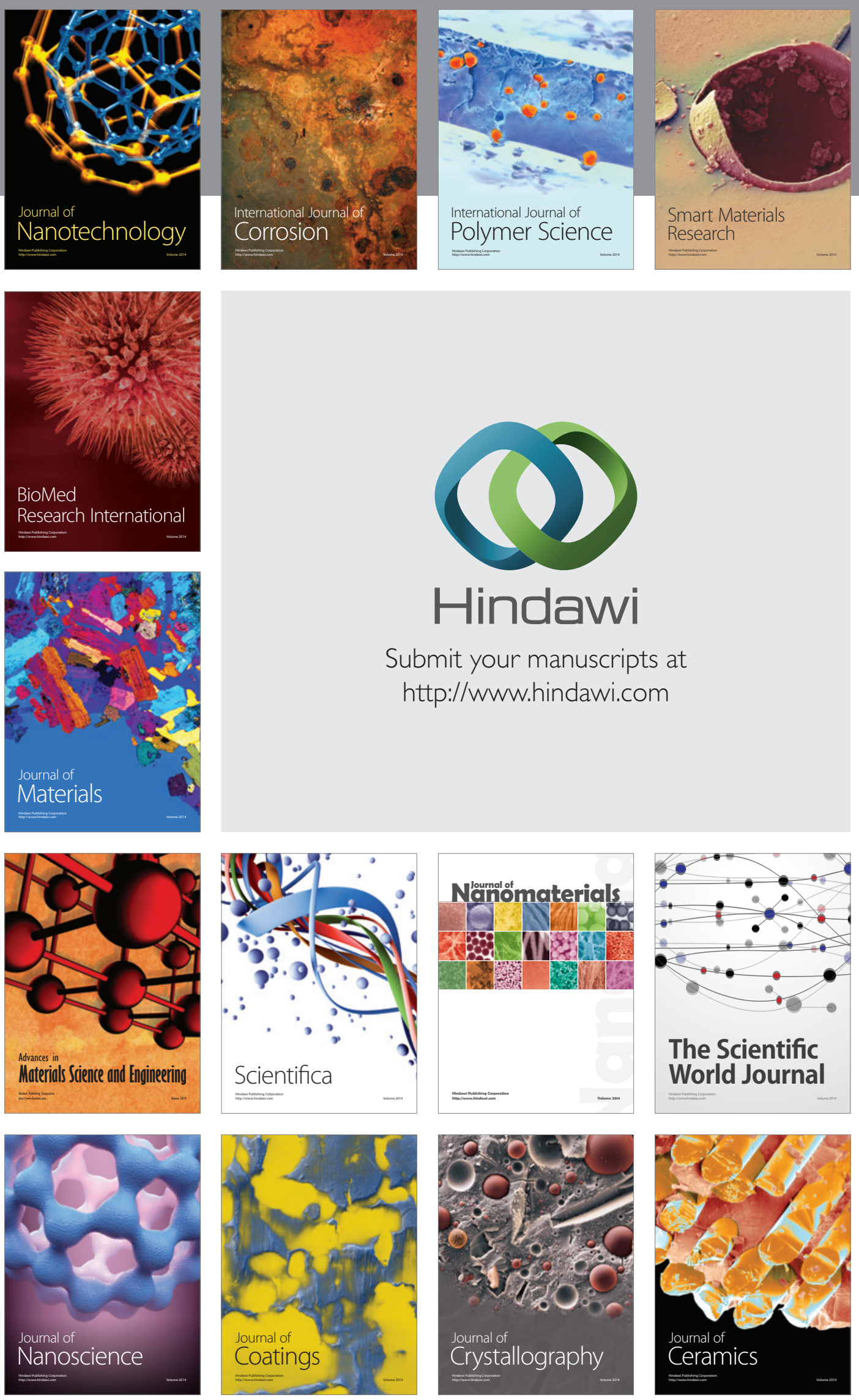

The Scientific World Journal

Submit your manuscripts at

http://www.hindawi.com

\section{World Journal}

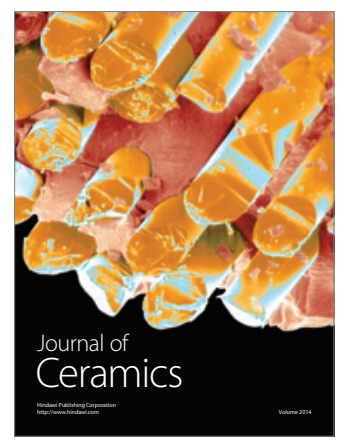

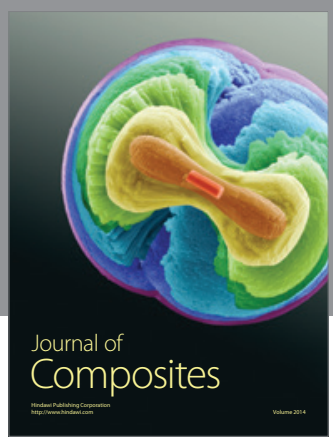
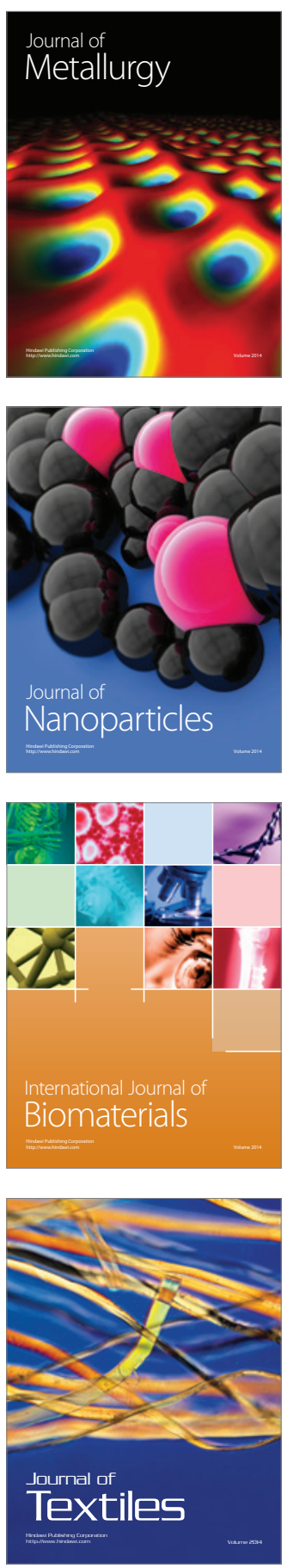